\title{
Parasite specialization from a phylogenetic perspective: a new index of host specificity
}

\author{
R. POULIN ${ }^{1 *}$ and D. MOUILLOT ${ }^{2}$ \\ ${ }^{1}$ Department of Zoology, University of Otago, P.O. Box 56, Dunedin, New Zealand \\ ${ }^{2}$ UMR CNRS-UMII 5119 Ecosystèmes Lagunaires, Université Montpellier II, CC 093, 34095 Montpellier cedex 5, France
}

(Received 5 October 2002; revised 26 November 2002; accepted 26 November 2002)

\section{SUMMARY}

The host specificity of a parasite is not merely a function of how many host species it can exploit, but also of how closely related these host species are to each other. Here, a new index of host specificity is proposed, one that takes into account the average taxonomic or phylogenetic distance between pairs of host species used by a parasite. The index is derived from measures of taxonomic distinctness used in biodiversity studies. It is easy to compute and interpret, ranging from a minimum value of 1 when all host species are members of the same genus, to a maximum of 5 , when all host species belong to different classes. The variance of this measure can also be computed, and provides additional information on the taxonomic or phylogenetic structure of the host assemblage. Using data on helminth parasites of Canadian freshwater fishes, we show that the new index, unlike the mere number of known host species, is independent of study effort i.e. the number of published records of a parasite. Although the index and the number of known hosts are not entirely independent statistically, each captures a different aspect of host specificity. For instance, although acanthocephalans infect significantly more host species than trematodes, cestodes or nematodes, there is no difference in the average index value among these 4 helminth taxa, suggesting that the average taxonomic distances between the host species of a parasite do not vary among these higher taxa. We recommend the use of our new index in future comparative studies of host specificity, in particular when the focus is on the evolutionary history of parasites and of their past colonizations of host lineages.

Key words: freshwater fishes, helminths, phylogenetic diversity, study effort, taxonomic distinctness.

\section{INTRODUCTION}

One of the most fundamental properties of any parasite species is its host specificity (Adamson \& Caira, 1994). In its most basic sense, the specificity of a parasite is determined by the number of host species it can exploit successfully (i.e. 'host range' sensu Lymbery, 1989). Although its measurement is not as straightforward as it may seem, this single feature of parasites is important for many reasons. For instance, it reflects, better than any other parameter, the breadth of their ecological niche, and thus their exact position and role in the biosphere. In addition, host specificity will determine the likelihood that a parasite will successfully invade a new habitat, or adjust to new hosts following its introduction to a new geographical area. Host specificity is therefore an important life-history trait of parasites, just as important if not more so than body size and fecundity. It has, however, proven extremely difficult to quantify in any way that would allow meaningful comparisons among species.

The main problem is that not all host species are equal. Two parasite species that each exploit

* Corresponding author: Department of Zoology, University of Otago, P.O. Box 56, Dunedin, New Zealand. Tel: +6434797983. Fax: +6434797584. E-mail: robert.poulin@stonebow.otago.ac.nz
4 host species at the corresponding stage of their life-cycles, say, as adults, may not be equally specialized. From an ecological perspective, parasite species A might be very common in 1 host species but rare in the other 3 , whereas parasite species $B$ might be equally prevalent and abundant in all 4 of its hosts. This pattern would suggest differences in host preferences or host exploitation. Rohde (1980, 1993, 2002) has developed indices of host specificity based on the relative intensities or prevalence of infection of a parasite in all its known host species. Despite some weaknesses (see Poulin, 1998), these indices at least take into account the relative importance of different host species as resources for parasites. Often, though, interest in host specificity is motivated by studies of host-parasite coevolution or by assessments of the risks of a parasite species colonizing a new host species, say, following a species introduction. If we now consider the above 2 hypothetical species of parasites from an evolutionary perspective, it is not the prevalence or intensity of infection that matters the most, but the phylogenetic relationships among the host species. If all 4 host species of parasite species A are congeners, whereas those of parasite species $\mathrm{B}$ all belong to different families, we have a situation in which one parasite (species A) is much more specific than the other because its hosts represent only a single host 
genus. Parasites with low host specificity are those characterized by broad taxonomic 'jumps' during their evolutionary history, regularly switching from one host species to a distantly related one. A useful measure of host specificity needs to take host relationships into account: the number of host species used on its own is inadequate for comparisons among different parasite species.

Recently, Caira, Jensen \& Holsinger (2003) proposed a new index of specificity that attempts to incorporate basic phylogenetic information on the host species used by a parasite. Their index considers not only how many host species are used by a parasite, but also how many genera, families and orders these hosts belong to. This is certainly a step in the right direction. This new index, however, is still not entirely satisfactory. Consider 2 parasite species, each parasitizing 10 host species representing 5 genera. In the first parasite species, 6 host species are congeners, and the other 4 each belong to a different genus. In the second parasite species, the 5 host genera each have 2 species exploited by the parasite. Caira et al.'s (2003) index would fail to discriminate between these 2 hypothetical parasite species, because it is not sensitive to the uneven distribution of host species among higher taxa. What we need is an index of host specificity that takes into account the finer phylogenetic or taxonomic structure of the group of host species used by a parasite.

Here, we present a new index that does just that. It is inspired from measures of phylogenetic diversity recently proposed in the scientific literature, in particular in biodiversity and conservation studies (e.g. Faith, 1992; Humphries, Williams \& VaneWright, 1995; Purvis \& Hector, 2000; Shimatani, 2001 ; Barker, 2002). More specifically, it is derived from the measures of taxonomic distinctness developed in recent studies of marine biodiversity by Clarke \& Warwick (1998, 1999, 2001; Warwick \& Clarke, 1998, 2001). We illustrate the use of this index with data on helminth parasites of Canadian freshwater fishes. We show that the new index has several properties that make it an unbiased estimator of the mean taxonomic distance between the hosts used by a parasite.

\section{METHODS}

\section{The new index}

The proposed specificity index, $S_{\mathrm{TD}}$, measures the average taxonomic distinctness of all host species used by a parasite species. When these host species are placed within a taxonomic hierarchy, based on the Linnean classification into phyla, classes, orders, families, genera and species, the average taxonomic distinctness is simply the mean number of steps up the hierarchy that must be taken to reach a taxon common to 2 host species, computed across all

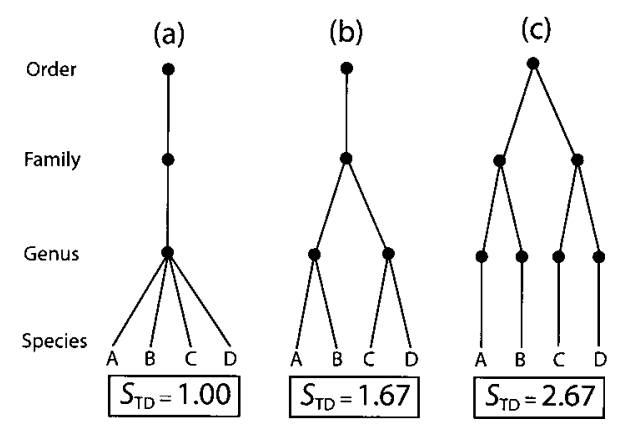

Fig. 1. Hypothetical taxonomic trees for the hosts of 3 species of parasites. Each parasite species is found on 4 host species, A-D. In (a), all 4 host species are congeners, and thus they average a single step toward a common node i.e. $S_{\mathrm{TD}}=1$. In (b), the 4 hosts belong to 2 different genera but to the same family, with some species pairs, such as $\mathrm{A}-\mathrm{B}$, requiring 1 step to reach a common node, and others, such as $\mathrm{A}-\mathrm{C}$, requiring 2 steps. Example (c) shows even greater taxonomic distinctness, with the 4 hosts belonging to different families within the same order. Note that the maximum possible value of $S_{\mathrm{TD}}$ in these examples is 3 , because there are 3 taxonomic levels above species.

possible pairs of host species (Fig. 1). Thus, if 2 host species are congeners, 1 step (species-to-genus) is necessary to reach a common node in the taxonomic tree; if the 2 species belong to different genera but the same family, 2 steps will be necessary (speciesto-genus, and genus-to-family); and so on, with these numbers of steps averaged across all host species pairs. For any given species pair, the number of steps corresponds to half the path length connecting two species in the taxonomic tree, with equal step lengths of one being postulated between each level in the taxonomic hierarchy. The greater the taxonomic distinctness between host species, the higher the number of steps needed, and the higher the value of the index $S_{\mathrm{TD}}$ : thus it is actually inversely proportional to specificity. A high index value means that on average the hosts of a parasite species are not closely related. Formally, the index is computed as follows (see Clarke \& Warwick, 1998):

$S_{\mathrm{TD}}=2 \frac{\sum \sum_{i<j} \omega_{i j}}{s(s-1)}$,

where $s$ is the number of host species used by a parasite, the double summation is over the set $\{i=1, \ldots s$; $j=1, \ldots s$, such that $i<j\}$, and $\omega_{i j}$ is the taxonomic distinctness between host species $i$ and $j$, or the number of taxonomic steps required to reach a node common to both (see Fig. 1).

Using the standard 5 taxonomic levels above species i.e. genus, family, order, class and phylum, the maximum value that the index $S_{\mathrm{TD}}$ can take (when all host species belong to different classes) is 5 , and its lowest value (when all host species are congeners) is 1 . Given that few (if any) parasite species, at a given stage in their life-cycle, infect hosts belonging 


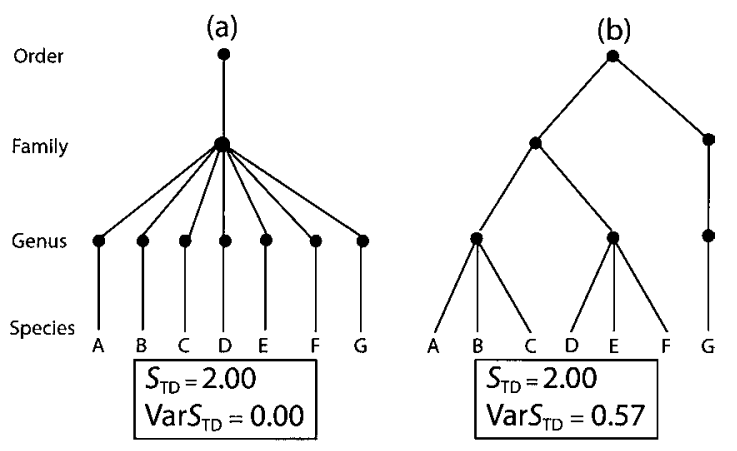

Fig. 2. Hypothetical taxonomic trees for the hosts of 2 species of parasites, in which both parasites exploit the same number of host species and have identical $S_{\mathrm{TD}}$ values. The greater asymmetry of the tree in (b), however, leads to a greater variance in taxonomic distinctness than in (a). In example (b), many hosts are congeners, but one belongs to a different family altogether, creating more variation in the number of steps required to reach the common node of pairs of species, than in example (a) where all host species belong to the same family (modified from Clarke \& Warwick, 2001).

to different phyla, this range is sufficient for all practical purposes, and we recommend it as a standard procedure.

It should be noted that the concept of taxonomic distinctness can easily be used in a full phylogenetic context, by replacing taxonomic trees such as those in Fig. 1 by proper phylogenetic trees with known branch lengths, with only a slight modification to the equation above (see Clarke $\&$ Warwick, 2001). However, given that we do not yet have fully-resolved phylogenies for all host taxa, it is probably more practical to stay within a taxonomic framework, at least at present. In addition, it is possible to incorporate ecological information, such as the prevalence or intensity of infection of the parasite in each of its host species, into the computations of taxonomic distinctness (Clarke \& Warwick, 1998; Barker, 2002). Again, however, such ecological data are not always available for all host species, especially when dealing with several parasite species in a comparative analysis; we therefore here focus on the simpler version of the index, based solely on the presence of the parasite on host species.

The index $S_{\mathrm{TD}}$ measures the average taxonomic distinctness between host species, and does not capture all of the taxonomic structure of a set of host species. It is possible to have 2 parasite species, each infecting the same number of host species and each characterized by an identical value of $S_{\mathrm{TD}}$, but with 1 parasite species clearly capable of infecting hosts across a broader taxonomic range (see Fig. 2). Asymmetries in the taxonomic distribution of host species across higher taxa can sometimes be missed by $S_{\mathrm{TD}}$, which is only the average taxonomic distinctness; in these situations complementary information can be obtained by examining the variance in taxonomic distinctness (see Clarke \& Warwick, 2001):

$\operatorname{Var}_{\mathrm{TD}}=\frac{\sum \sum_{i \neq j}\left(\omega_{i j}-\varpi\right)^{2}}{s(s-1)}$

where $\varpi$ is simply the average taxonomic distinctness, or $S_{\mathrm{TD}}$. The variance in $S_{\mathrm{TD}}$ is not as informative as $S_{\mathrm{TD}}$ itself, and not as convenient as an index, but it conveys separate information of how much taxonomic heterogeneity there is among a group of host species. In other words, $\operatorname{Var}_{\mathrm{TD}}$ measures the 'evenness' of the distribution of taxa across the taxonomic tree (Warwick \& Clarke, 2001) or the 'complexitiy' of this hierarchical tree. Note, however, that the variance in $S_{\mathrm{TD}}$ can only be computed when a parasite exploits 3 or more host species (it always equals zero with 2 host species).

The only apparent weakness of $S_{\mathrm{TD}}$ is that it cannot be applied to parasite species infecting only 1 host species. There is no pair of host species in these cases from which an average taxonomic distance can be computed. There are 2 obvious ways of dealing with such highly host-specific parasites. First, they can be excluded from any comparative analysis in which $S_{\mathrm{TD}}$ is computed for other, less specific parasite species; after all, they show maximum specificity, and the computation of an index will not tell us more about them. Second, they can be assigned a $S_{\mathrm{TD}}$ value of 1 , since by definition 'all' host species for such specialized parasites belong to the same genus. This option has merits when it is the host taxonomic level beyond which parasites do not colonize new hosts that is of interest, whereas the former option is preferable when one is interested in the taxonomic extent of host switching in parasites that have actually achieved switches.

\section{Data and analyses}

We obtained data on the host fish species used by species of trematodes, cestodes, nematodes and acanthocephalans parasitizing Canadian freshwater fishes. The data were obtained from the checklists of Margolis \& Arthur (1979). For certain parasite groups, there are more up-to-date host lists (e.g. for trematodes, see Gibson, 1996), and revisions of helminth taxonomy may have affected the species status of a few helminths included in our analyses, but these would have little impact on the results as a whole. Besides, these data are used only to illustrate the usefulness of the new index.

Data were gathered only for parasite species that use fish as definitive hosts, i.e. that occur in fish as adult worms. Number of known host species were recorded for each of these helminth species. In addition, we computed our new index of host taxonomic distinctness, $S_{\mathrm{TD}}$ (only for parasite species with more than 1 known host species), as well as its variance (only for parasite species with more than 


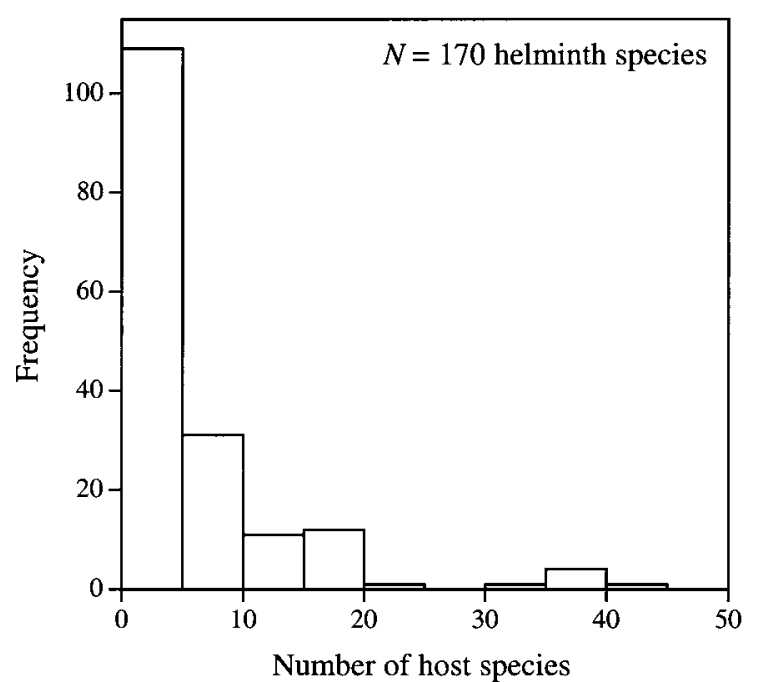

Fig. 3. Frequency distribution of known number of host species among helminth species parasitic on Canadian freshwater fishes.

2 known hosts) for these helminth species. To calculate the index and its variance, a computer program was developed using borland $\mathrm{C}++$ Builder 5.0. The fish taxonomy used for the calculations was that proposed by Nelson (1994), because his comprehensive taxonomic hierarchy is based on a phylogenetic scheme; other phylogenies/taxonomies could have been used, however. We used the 6 taxonomic levels (species, genus, family, order, class, and phylum) recommended above; these were necessary anyway because some of the helminths infect both fish and lampreys, which only come together at the phylum level. Finally, we also recorded for each helminth species the number of published records of its occurrence. This variable serves as a measure of study effort. A parasite only recorded once is likely to have been found on a single host species, regardless of how many it actually parasitizes; the number of known host species is therefore positively related to study effort, or how often a particular parasite has been recorded (see Poulin, 1992). This confounding variable was thus taken into account in our analyses.

Data on number of known host species and number of published records were log-transformed prior to analysis using standard parametric tests, even if raw values are sometimes reported in tables or figures.

\section{RESULTS}

We obtained data for a total of 170 species of helminths parasitic in Canadian fishes. Of these, 108 species had at least 2 host species and could be assigned a $S_{\mathrm{TD}}$ value. The distribution of numbers of known host species was clearly skewed, with most helminth species being found in only one or two host species (Fig. 3). The distribution of $S_{\text {TD }}$ values was closer to normal, with a modal value of just
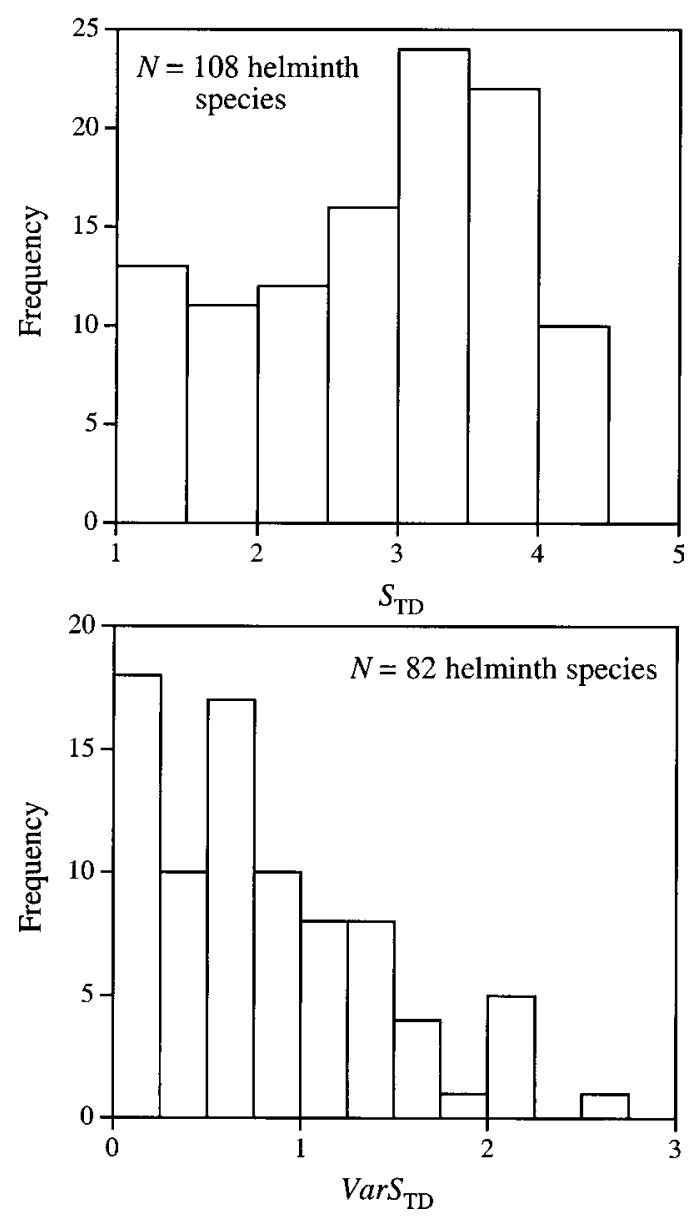

Fig. 4. Frequency distribution of $S_{\mathrm{TD}}$ and $\operatorname{Var} S_{\mathrm{TD}}$ values among helminth species parasitic on Canadian freshwater fishes.

over 3 (Fig. 4). This indicates that a typical helminth of freshwater fish infects host species belonging to different orders. The frequency distribution of $\operatorname{Var}_{\mathrm{TD}}$ values (Fig. 4) shows that most are small, and thus that highly asymmetrical patterns of host relationships such as that in Fig. $2 \mathrm{~B}$ are rather uncommon.

The number of known host species correlated positively with the number of published records across all helminth species $(r=0 \cdot 716, N=170, P=0 \cdot 0001)$ : the more is published about a parasite species, the longer its list of known hosts is likely to be (Fig. 5). The same was not true for either $S_{\mathrm{TD}}(r=0 \cdot 146$, $N=108, P=0 \cdot 1306)$ or $\operatorname{Var}_{\mathrm{TD}}(r=0 \cdot 103, N=82$, $P=0 \cdot 3560)$ across helminth species for which these indices could be computed (Fig. 5). Both these measures are apparently insensitive to study effort, making them less biased indices of host specificity than just the number of known host species.

The index $S_{\mathrm{TD}}$ is not entirely independent of the number of known host species, however: the 2 measures are positively correlated $(r=0.446$, $N=108, P=0 \cdot 0001)$. The roughly triangular scatter of points (Fig. 6) suggests that although both low and high values of $S_{\mathrm{TD}}$ are possible when few host 


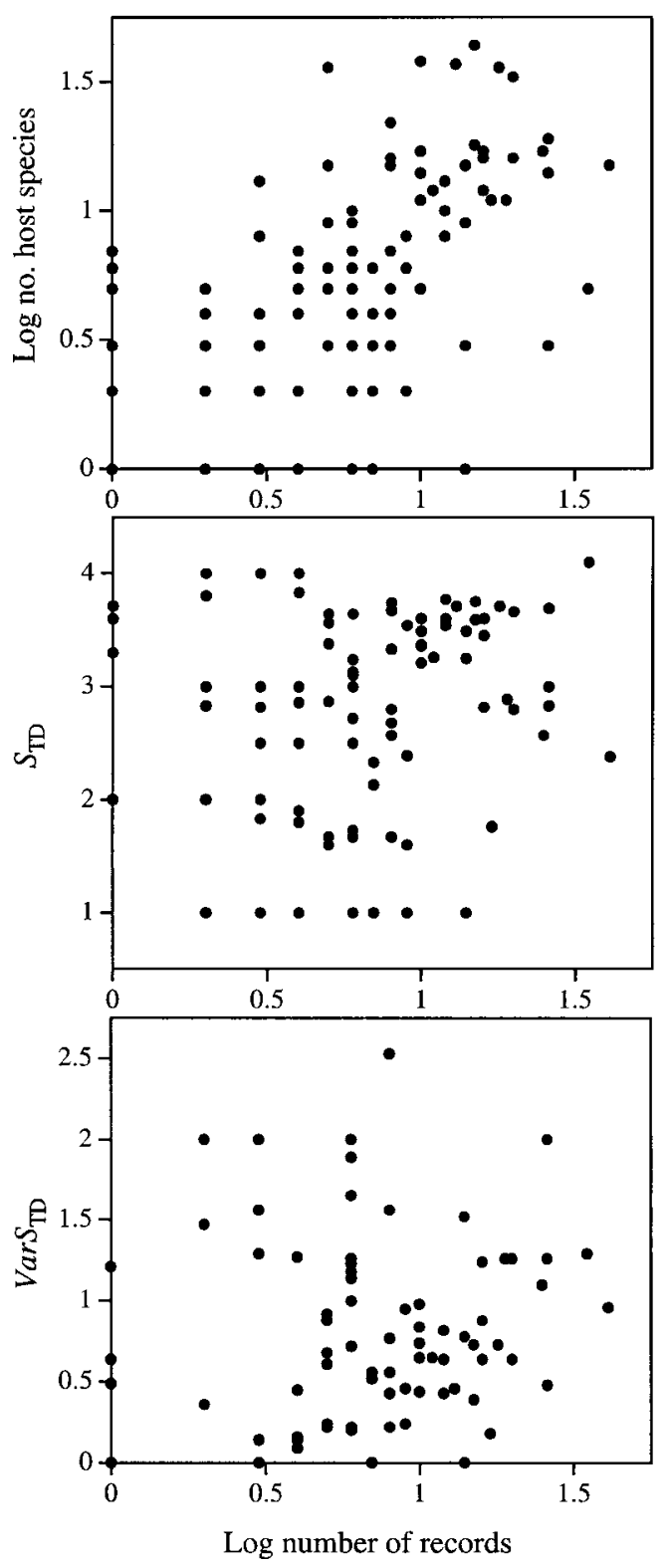

Fig. 5. Relationships between the number of published records of each helminth species found in Canadian freshwater fishes, and 3 measures of their host specificity: the number of known host species per parasite species, $S_{\mathrm{TD}}$ and $\operatorname{Var} S_{\mathrm{TD}}$.

species are known, low values of $S_{\mathrm{TD}}$ become increasingly less likely for parasites with many hosts, probably because these parasites are more likely to include hosts from a broader taxonomic origin. Even after correcting the number of known hosts for study effort, by using the residuals of a regression against the number of published records, the correlation with $S_{\mathrm{TD}}$ remains significant $(r=0 \cdot 403, N=108$, $P=0.0001)$. In contrast, $\operatorname{Var} S_{\mathrm{TD}}$ does not covary with the number of known hosts $(r=0 \cdot 002, N=82$, $P=0.9891$ ), even after a correction for study effort $(r=-0 \cdot 098, N=82, P=0 \cdot 3787)$. It appears, however, that $\operatorname{Var}_{\mathrm{TD}}$ can achieve a wider range of values in parasites with few known hosts than in parasites with many known host species (Fig. 6).

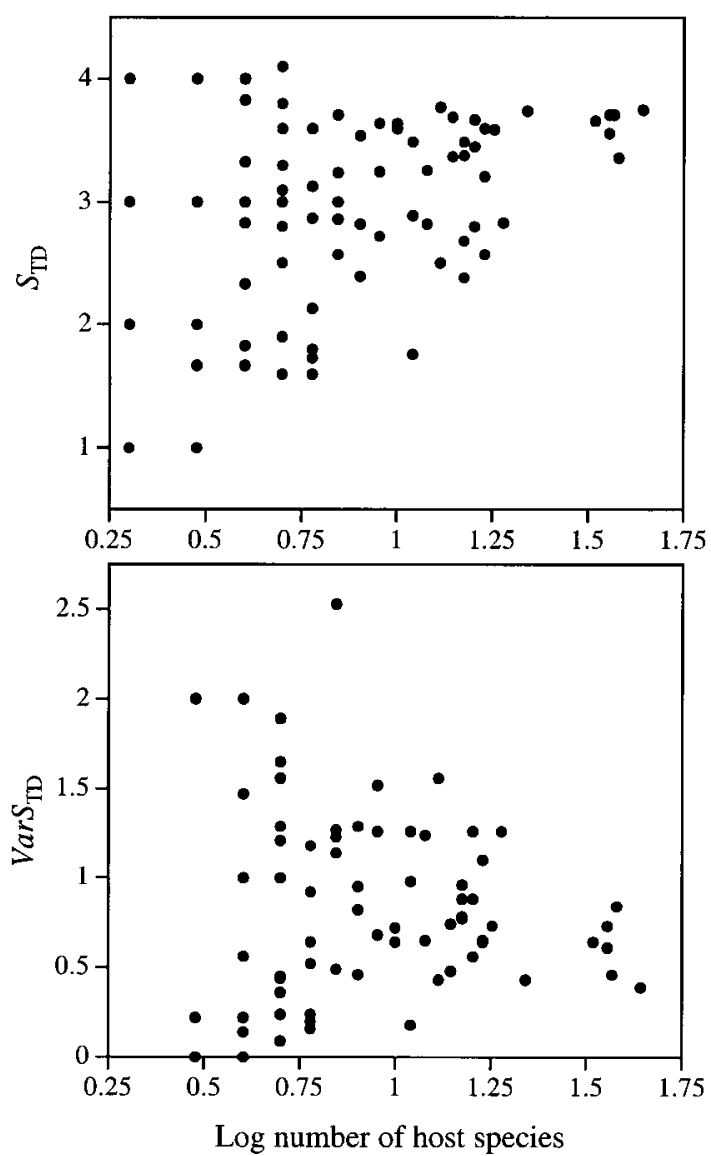

Fig. 6. Relationships between the number of known host species per helminth species and both $S_{\mathrm{TD}}$ and $\operatorname{Var} S_{\mathrm{TD}}$, across helminth parasites of Canadian freshwater fishes.

There is also a weak positive correlation between $S_{\mathrm{TD}}$ and $\operatorname{Var}_{\mathrm{TD}}(r=0 \cdot 213, N=82, P=0.0552)$. This is not too surprising, as the variance and mean of a group of numbers are never independent, but it also means that these two indices capture some of the same features of a taxonomic assemblage of host species.

Comparisons among the 4 helminth taxa (trematodes, cestodes, nematodes and acanthocephalans) are also revealing. Although they do not differ with respect to study effort, the 4 taxa have different numbers of known host species (Table 1). On average, acanthocephalan species exploit twice as many host species as nematodes do, and 3 times more host species than trematodes or cestodes. However, the 4 taxa do not differ significantly with respect to $S_{\mathrm{TD}}$ or $\operatorname{Var} S_{\mathrm{TD}}$ (Table 1). This means that although acanthocephalans colonize more new host species than other helminths, they do not make bigger taxonomic 'jumps' when they switch hosts.

\section{DISCUSSION}

Recent interest in host-parasite coevolution has followed in the footsteps of progress in phylogenetic reconstructions of the evolutionary history of related 
Table 1. Comparisons of study effort and 3 measures of host specificity (means \pm s.D.) among 4 large taxa of helminths parasitizing Canadian freshwater fishes

\begin{tabular}{|c|c|c|c|c|c|}
\hline Parasite taxon & $N$ & $\begin{array}{l}\text { No. of records } \\
\text { /species }\end{array}$ & $\begin{array}{l}\text { No. of host } \\
\text { species }\end{array}$ & $S_{\mathrm{TD}}$ & $\operatorname{Var} S_{\mathrm{TD}}$ \\
\hline Trematodes & 63 & $4 \cdot 76(5 \cdot 03)$ & $4 \cdot 16(4 \cdot 58)$ & $2 \cdot 69(1 \cdot 03)$ & $0 \cdot 69(0 \cdot 46)$ \\
\hline Cestodes & 50 & $6.92(8.52)$ & $4 \cdot 01(4 \cdot 21)$ & $2 \cdot 66(0 \cdot 94)$ & $0 \cdot 87(0 \cdot 73)$ \\
\hline Nematodes & 39 & $5 \cdot 49(5 \cdot 45)$ & $6 \cdot 85(9 \cdot 12)$ & $2 \cdot 82(0 \cdot 84)$ & $0 \cdot 82(0 \cdot 56)$ \\
\hline Acanthocephalans & 18 & $7 \cdot 78(6 \cdot 26)$ & $12 \cdot 61(14 \cdot 41)$ & $3 \cdot 05(0.92)$ & $0 \cdot 94(0 \cdot 53)$ \\
\hline ANOVAs & & $\begin{array}{l}F_{3,166}=1 \cdot 195 \\
P=0 \cdot 3133\end{array}$ & $\begin{array}{l}F_{3,166}=4 \cdot 017 \\
P=0 \cdot 0086\end{array}$ & $\begin{array}{l}F_{3,104}=0 \cdot 668 \\
P=0.5738\end{array}$ & $\begin{array}{l}F_{3,78}=0 \cdot 721 \\
P=0 \cdot 5424\end{array}$ \\
\hline
\end{tabular}

groups of animals (see Brooks \& McLennan, 1993; Paterson \& Gray, 1997; Jackson, 1999). This emphasis on phylogenetic relationships should be reflected in any measure of host specificity, because the host specificity of parasites is a simple concept encompassing their evolutionary history of associations with different host taxa (Caira et al. 2003). Here, we present a simple measure of host specificity that quantifies precisely the average relatedness of the host species used by a parasite. A single value cannot capture all the nuances associated with the concept of host specificity. From an evolutionary perspective, however, our new index provides a brief summary of a parasite's history of host switching, and of the average taxonomic (or phylogenetic) distance between hosts during host switches.

Not only is the new index providing an accurate assessment of host relatedness, but it also possesses other desirable features. First, it is independent of study effort, just as the index used in biodiversity studies from which $S_{\mathrm{TD}}$ is derived is independent of sample size (Rogers, Clarke \& Reynolds, 1999; Clarke \& Warwick, 1999, 2001; Warwick \& Clarke, 1998, 2001). The strong link between study effort and the number of recorded host species has plagued past attempts to use this simple count as a measure of host specificity (Poulin, 1992). Our new index is not affected by this problem. Second, although $S_{\mathrm{TD}}$ does correlate with the number of known host species, the two parameters clearly measure different things. This is illustrated clearly in the comparisons of the values for the four helminth groups. Despite showing much longer lists of known host species, on average, than other helminth taxa, the acanthocephalans had values of $S_{\mathrm{TD}}$ or $\operatorname{Var} S_{\mathrm{TD}}$ similar to those of trematodes, cestodes and nematodes. Using our index sheds new light on these comparisons, and suggests that there are no real differences in host specificity among the four helminth taxa. Acanthocephalans might be more likely to colonize new host species, but in doing so they do not make longer taxonomic 'jumps' than other helminths.

The usefulness of the index, and its ability to distinguish between parasite species that exploit the same number of host species, can be best seen in specific examples. Consider the trematodes Homalometron armatum and Acetodextra amiuri. Both have four known host species according to Margolis \& Arthur (1979), including the bullhead Ictalurus nebulosus on which both trematodes are found. However, whereas the other 3 fish hosts of $A$. amiuri are also all in the family Ictaluridae, those of $H$. armatum belong to different orders (Siluriformes, Perciformes, and Acipenseriformes). This is reflected in the $S_{\mathrm{TD}}$ values of $A$. amiuri and $H$. armatum, which are 1.67 and 3.83 , respectively. Only $S_{\mathrm{TD}}$ can discriminate quickly and quantitatively between parasite species like these, i.e. parasites with equal numbers of host species but with very different colonizing abilities.

Alternatively, $S_{\mathrm{TD}}$ can help to see through apparent differences in host specificity that are based solely on the number of known host species. For example, consider the nematodes Philometra cylindracea and Rhabdochona cascadilla. According to Margolis \& Arthur (1979), the former occurs in 4 host species and the latter in 38 host species, despite both species having received similar study effort ( 8 and 10 published surveys, respectively, mentioning these parasite species). Despite this marked difference, however, their $S_{\mathrm{TD}}$ values are practically identical : $3 \cdot 33$ and $3 \cdot 36$, respectively. These 2 nematode species have therefore crossed very similar taxonomic distances when they colonized new host species over their evolutionary history. One species, $R$. cascadilla, has made more such colonizations than the other, as shown by its longer list of known hosts, and that is where the difference lies. This difference may simply be due to contrasting opportunities for colonisation: half of the hosts of $R$. cascadilla are cyprinids, by far the most speciose family of Canadian freshwater fishes, whereas $P$. cylindracea does not infect any cyprinid. Exploiting hosts belonging to speciose taxa promotes host switching among related host species (Poulin, 1992). The number of known hosts and $S_{\mathrm{TD}}$ convey information about different aspects of a parasite's history of host colonization. Both are easy to compute, and they should both be used in future comparative analyses of large numbers of parasite species in order to elucidate 
which factors contribute to both the frequency of host switches, and the relative taxonomic distances involved in those switches.

The variance in $S_{\mathrm{TD}}$, or $\operatorname{Var} S_{\mathrm{TD}}$, also provides useful information, but is not as readily useful as $S_{\text {TD }}$ itself. A high variance usually means that one main branch in the taxonomic tree of host species contributes proportionally more species to the list than other branches, but the exact value of $\operatorname{Var} S_{\mathrm{TD}}$ does not lend itself to easy interpretation. In contrast, values for $S_{\mathrm{TD}}$ have a simple biological meaning. An $S_{\mathrm{TD}}$ value of $1 \cdot 1$ means that most of the known host species of a parasite are congeners, a value of 1.8 means that they belong to the same family but are mostly from different genera, a value of 2.5 indicates that several of them belong to different orders, and so on. We therefore recommend $S_{\mathrm{TD}}$ as an index of specificity, but encourage the use of $\operatorname{Var} S_{\mathrm{TD}}$ as a source of additional information about the taxonomic structure, complexity or unevenness of the host assemblage (see Clarke \& Warwick, 2001).

Our new index emphasizes phylogenetic or taxonomic distances between host species, but it ignores ecological distance. It is conceivable that 2 closely related host species diverge with respect to one or more ecological features important for parasite colonization, such as host diet or body size, whereas 2 more distantly-related host species may live in sympatry and share several ecological features. In such cases, the ecological distance between hosts becomes the main determinant of the likelihood of host switching, with phylogenetic distance playing a secondary role. Ecological distances are more difficult to quantify than phylogenetic or taxonomic distances, however, and cannot easily be integrated into an index of host specificity. In addition, phylogenetic and ecological distances are likely to go hand in hand, in most situations, such that members of the same genus tend to have similar body sizes, diets, habitat preferences, etc. Another aspect of specificity not integrated into our index is that of microhabitat specificity. Two parasite species may exploit the same 3 host species, with one found everywhere in the gut and the other restricted to a very short section of the gut. The latter parasite is therefore more specialized than the former, but on a scale not covered by our index.

In conclusion, we present a new index of host specificity that measures the average taxonomic distances between all the host species used by a parasite. The new index is easy to compute, independent of sampling effort, and it conveys information that is not provided by the mere number of host species known to be exploited by a parasite. An even better index would be one that incorporates ecological information such as the prevalence or abundance of the parasite in each of its host species. This could be achieved by weighing the different branches of the host taxonomic tree according to prevalence or abundance; given the general lack of good ecological data for many parasite species, the usefulness of this more complete version of the index would be limited. The version of $S_{\mathrm{TD}}$ presented here is likely to be the one with the broadest application. Similar indices, from which the present one is derived, have been used in biodiversity studies and have provided new light on the conservation value of certain natural assemblages, or on the impact of anthropogenic disturbances on biodiversity (e.g. Warwick \& Clarke, 1995, 1998; Rogers et al. 1999; Purvis \& Hector, 2000; von Euler \& Svensson, 2001; Barker, 2002). These new indices have shifted the emphasis from the mere number of species in a locality to the diversity of the higher taxa that they represent. Applied to the specificity of parasites for their hosts, our new index can do the same thing, and become a powerful tool in future studies of parasite specialisation.

We thank Tine Huyse and two anonymous referees for constructive comments on an earlier version of the manuscript. R.P. is supported by a James Cook Research Fellowship from the Royal Society of New Zealand.

\section{REFERENCES}

ADAMSON, M. L. \& CAIRA, J. N. (1994). Evolutionary factors influencing the nature of parasite specificity. Parasitology 109, S85-S95.

BARKer, G. M. (2002). Phylogenetic diversity: a quantitative framework for measurement of priority and achievement in biodiversity conservation. Biological Fournal of the Linnean Society 76, 165-194.

BRoOKs, D. R. \& Mclennan, D. A. (1993). Parascript: Parasites and the Language of Evolution. Smithsonian Institution Press, Washington, D.C.

CAIRA, J. N., JENSEN, K. \& HOLSINGER, K. E. (2003). On a new index of host specificity. In Taxonomy, Ecology and Evolution of Metazoan Parasites (ed. Combes, C. \& Jourdane, J.), Presses Universitaires de Perpignan, Perpignan, France (in the Press).

Clarke, K. R. \& WARWICK, R. M. (1998). A taxonomic distinctness index and its statistical properties. Fournal of Applied Ecology 35, 523-531.

CLARKE, K. R. \& WARWICK, R. M. (1999). The taxonomic distinctness measure of biodiversity: weighting of step lengths between hierarchical levels. Marine Ecology Progress Series 184, 21-29.

ClARKE, K. R. \& WARWICK, R. M. (2001). A further biodiversity index applicable to species lists: variation in taxonomic distinctness. Marine Ecology Progress Series 216, 265-278.

FAITH, D. P. (1992). Conservation evaluation and phylogenetic diversity. Biological Conservation 61, 1-10. GIBSON, D. I. (1996). Guide to the parasites of fishes of Canada. Part IV: Trematoda. Canadian Special Publication of Fisheries and Aquatic Sciences 124, 1-373. HUMPHRIES, C. J., WILLIAMS, P. H. \& VANE-WRIGHT, R. I. (1995). Measuring biodiversity value for conservation. Annual Review of Ecology and Systematics 26, 93-111. 
JACKSON, J. A. (1999). Analysis of parasite host-switching: limitations on the use of phylogenies. Parasitology 119, S111-S123.

LYMBERY, A. J. (1989). Host specificity, host range and host preference. Parasitology Today 5, 298.

MARGOLIS, L. \& ARTHUR, J. R. (1979). Synopsis of the

Parasites of Fishes of Canada. Bulletin of the Fisheries Board of Canada 199, Ottawa, Canada.

NELson, J.s. (1994). Fishes of the World, 3rd Edn. John Wiley \& Sons, New York.

PATERSON, A. M. \& GRAY, R. D. (1997). Host-parasite cospeciation, host switching and missing the boat. In Host-Parasite Evolution: General Principles and Avian Models (ed. Clayton, D. H. \& Moore, J.), pp. 236-250. Oxford University Press, Oxford.

POULIN, R. (1992). Determinants of host specificity in parasites of freshwater fishes. International Yournal for Parasitology 22, 753-758.

POUlin, R. (1998). Evolutionary Ecology of Parasites : From Individuals to Communities. Chapman and Hall, London. PURVIS, A. \& HECTOR, A. (2000). Getting the measure of biodiversity. Nature, London 405, 212-219.

ROGERS, S. I., CLARKE, K. R. \& REYNOLDS, J. D. (1999). The taxonomic distinctness of coastal bottom-dwelling fish communities of the North-east Atlantic. Fournal of Animal Ecology 68, 769-782.
ROHDE, K. (1980). Host specificity indices of parasites and their application. Experientia 36, 1369-1371.

RoHde, K. (1993). Ecology of Marine Parasites, 2nd Edn. CAB International, Wallingford, U.K.

ROHDE, K. (2002). Ecology and biogeography of marine parasites. Advances in Marine Biology 43, $1-86$.

Shimatani, K. (2001). On the measurement of species diversity incorporating species differences. Oikos 93, 135-147.

VON EULER, F. \& SVEnsson, s. (2001). Taxonomic distinctness and species richness as measures of functional structure in bird assemblages. Oecologia 129, 304-311.

WARWICK, R. M. \& ClARKE, K. R. (1995). New 'biodiversity' measures reveal a decrease in taxonomic distinctness with increasing stress. Marine Ecology Progress Series 129, 301-305.

WARWICK, R. M. \& ClARKE, K. R. (1998). Taxonomic distinctness and environmental assessment. Fournal of Applied Ecology 35, 532-543.

WARWICK, R. M. \& ClARKE, K. R. (2001). Practical measures of marine biodiversity based on relatedness of species. Oceanography and Marine Biology Annual Review 39, 207-231. 